\title{
GIANT CELL ARTERITIS (GCA) AND TAKAYASU ARTERITIS (TAK): A CASE REPORT ABOUT THE DIFFICULTY OF CLASSIFYING VASCULITIS THAT SHARE SIMILAR CHARACTERISTICS OF DISEASE.
}

Pedro Felipe de Almeida Vianna (Universidade Estadual do Rio de Janeiro, Rio de Janeiro, RJ, Brasil),

Camilla de Castro e Silva (Universidade Estadual do Rio de Janeiro, Rio de Janeiro, RJ, Brasil), Laissa Cristina Alves Alvino (Universidade Estadual do Rio de Janeiro, Rio de Janeiro, RJ, Brasil), Camila Souto Oliveira Elias (Universidade Estadual do Rio de Janeiro, Rio de Janeiro, RJ, Brasil), Manuella Lima Gomes Ochtrop (Universidade Estadual do Rio de Janeiro, Rio de Janeiro, RJ, Brasil)

\section{BACKGROUND}

Giant cell arteritis (GCA) and Takayasu arteritis (TAK) are granulomatous large-vessel vasculitides that share many common features. The American College of Rheumatology (ACR) classification criteria use age cut-offs for both conditions: age at disease onset $\leq 40$ years to classify TAK and age $\geq 50$ years to classify GCA. However, there are patients with GCA who present with upper extremity arterial insufficiency and large artery ischemic manifestations indistinguishable from TAK. Patients with large-vessel vasculitides who fall into the age range of 41-49 years are not included in the classification criteria for either condition.

\section{CASE REPORT}

We present a case report of a female patient who, at age 45 , initiated constitutional symptoms, such as fever, weight loss, associated with left upper limb claudication, arterial hypertension and asymmetry of peripheral pulses. Complementary imaging studies showed caliber reduction and thickening of the left subclavian artery wall after emergence of the vertebral artery. On that occasion she was diagnosed with Takayasu's arteritis and was followed up at the Rheumatology department of a University Hospital. Even in the presence of methotrexate, in January 2019, at age of 64 years, she developed a constant pattern temporal headache, worse at night and in the morning, accompanied by scalp tenderness, low daily fever and visual turbidity. There was additionally an elevation of ESR (25 to $60 \mathrm{~mm}$ ) and duplex ultrasound revealed an incompressible right superficial temporal artery with stenosis caused by concentric parietal thickening and reduced lumen. Ophthalmologic evaluation ruled out inflammatory vascular involvement as a cause of visual alteration. Treatment with prednisone $60 \mathrm{mg}$ daily was initiated leading to clinical improvement of headache, absence of fever, besides the significant reduction of ESR (60 to $10 \mathrm{~mm}$ ). With this case we question the diagnosis initially conferred to the patient, referring, this time, as giant cell arteritis.

\section{CONCLUSION}

GCA and TAK are large-vessel vasculitides that share clinical, histopathologic and radiographic features. To classify this patients can be a challenge, mainly between 41 to 49 years. A subset of patients with GCA can present with large artery ischemic manifestations like limb claudication, bruits and absent or diminished pulses and studies have found that these patients tend to be younger and have fewer cranial symptoms. We presented a case report of a female patient initially diagnosed as TAK presenting later with a temporal arteritis exemplifying this interesting condition and reinforcing the importance of close followup of patients with large vessels vasculitides. 